\title{
A new compact neutron/gamma ray scintillation detector
}

\author{
A. Buffler ${ }^{*}$ and A.C. Comrie ${ }^{\dagger}$ \\ Department of Physics, University of Cape Town, \\ Rondebosch, 7700, South Africa \\ *andy.buffler@uct.ac.za; ${ }^{\dagger}$ accomrie@gmail.com \\ F.D. Smit \\ iThemba LABS, National Research Foundation, \\ Somerset West, 7129, South Africa \\ smit@tlabs.ac.za \\ H.J. Wörtche \\ INCAS $S^{3}$ \\ Dr. Nassaulaan 9. 9400 AT Assen, The Netherlands; and \\ Department of Electrical Engineering, Eindhoven University of Technology \\ 600 MB Eindhoven, The Netherlands \\ heinrichwoertche@incas3.eu \\ Published 1 September 2016
}

\begin{abstract}
Progress towards the realization of a new compact neutron spectrometer is described. The detector is based on EJ299-33 plastic scintillator coupled to silicon photomultipliers, and a digital implementation of pulse shape discrimination is used to separate events associated with neutrons from those associated with gamma rays. The spectrometer will be suitable over the neutron energy range $1-100 \mathrm{MeV}$, illustrated in this work with measurements made using an AmBe radioisotopic source and quasi-monoenergetic neutron beams produced using a cyclotron.
\end{abstract}

Keywords: Neutron spectrometer; EJ299-33 scintillator; pulse shape discrimination.

PACS numbers: 29.30.Hs, 29.40.Mc.

\section{Introduction}

There is an ongoing need to develop compact neutron spectrometers, particularly for deployment in aircraft and spacecraft, for minerals exploration, for personal and workplace dosimetry and in security, particularly for the detection of special nuclear materials. Furthermore, for dosimetry in the upper atmosphere and in space, neutrons in the energy range $1-100 \mathrm{MeV}$ need to be measured. ${ }^{1}$

This is an Open Access article published by World Scientific Publishing Company. It is distributed under the terms of the Creative Commons Attribution 3.0 (CC-BY) License. Further distribution of this work is permitted, provided the original work is properly cited. 
Neutrons and gamma rays nearly always co-exist in the same radiation field; therefore, any useful detector will need to discriminate between the two radiation types. Organic scintillation detectors remain strong candidates for these applications because the light output of certain organic scintillators display both fast and slow decay-components, related to the specific energy loss of the recoiling charged particle by the Birks relation. ${ }^{2}$ Pulse shape discrimination (PSD), established ${ }^{3}$ in the 1950 s and developed through the 1960s, allows the identification of different types of charged particles in these scintillator detectors by means of the characteristics of the scintillation decay. Liquid organic scintillators such as EJ301 remain a popular choice when good-quality PSD is required.

Three recent technological developments have assisted with the development of detectors requiring PSD for use in non-laboratory environments. The invention of solid ("plastic") scintillators ${ }^{4,5}$ which exhibit PSD capability, such as the EJ299-33 scintillator used in the present work, remove the toxic and fire hazards associated with most liquid scintillators. Furthermore, plastic scintillators have no need for an optical window when coupled to a photodetector and may be easily produced in any geometrical form. Secondly, the development of efficient and compact silicon photomultipliers (SiPMs) offers a compact and robust alternative to traditional photomultiplier tubes and removes the requirement of high voltage. Finally, the emergence of digital data acquisition and processing systems allows pulse shape discrimination to be implemented and dynamically optimized in software across a wide energy range ${ }^{6-8}$ or implemented on specialized hardware, such as field programmable gate arrays (FPGAs) or digital signal processors (DSPs).

We are well-advanced in developing a compact neutron spectrometer, suitable for measurements between 1 and $100 \mathrm{MeV}$. The spectrometer comprises an EJ299-33 scintillator, SiPMs and an FPGA-based acquisition system. Detectors based on EJ299-33 scintillator have been well-characterized up to around $15 \mathrm{MeV} .{ }^{9.10}$ As the neutron energy increases, the proportion of recoiling charged particles that escape before full-energy deposition increases, which distorts the response function of the spectrometer. We present measurements of the response of EJ299-33 scintillators to neutrons and gamma rays from an $\mathrm{AmBe}$ source and from a cyclotron beam. Additionally, we illustrate the use of a small EJ299-33-based detector for spectrometry by unfolding measured pulse height spectra using a response matrix calculated via a GEANT4-based simulation.

\section{Experiments}

Measurements using two EJ299-33 scintillating detectors are presented. The reference detector is a cylindrical scintillator $(50 \mathrm{~mm}$ diameter $\times 50 \mathrm{~mm})$, optically coupled to an ETL 9214 12-stage photomultiplier tube (PMT) and base, supplied by Scionix, and operated at a negative bias of $950 \mathrm{~V}$. The second detector is $6 \times 6 \times 50 \mathrm{~mm}^{3}$ with each end coupled to a SensL MicroFC-60035 $\mathrm{SiPM}^{11}$ using silicone grease. The SiPMs were operated at $3.5 \mathrm{~V}$ above the breakdown voltage of $24.6 \mathrm{~V}$.

Signals from the anode of the PMT or the anode of each of the two SiPMs were digitized by a CAEN Vx1761 10 bit digitizer, operating at $4 \mathrm{GS} / \mathrm{s}$ with a peak-to-peak 
range of $1 \mathrm{~V}$. Custom-built software was used to record data for offline analyses. The total signal of the two SiPMs was taken as a geometric mean of the individual signals. This mitigated variation in light output due to variations in neutron interaction position in the scintillator and variation in gain between the two SiPMs.

Neutrons and gamma rays were provided via two methods: a $2 \mathrm{GBq}^{241} \mathrm{Am}^{9} \mathrm{Be}$ $(\mathrm{AmBe})$ radioisotopic source (producing about $1.4 \times 10^{5}$ neutrons $\mathrm{s}^{-1}$ ) and proton beams produced at the cyclotron facility of iThemba LABS, South Africa. The AmBe source produces $4.43 \mathrm{MeV}$ gamma rays as well as neutrons with a complex energy spectrum with maximum energy at about $10 \mathrm{MeV}$.

\section{Pulse Shape Discrimination}

Figure 1 shows typical digitized pulses for both detectors, each with the same total integral. The measurements were produced from $\mathrm{AmBe}$ where interactions of neutron and gamma rays in each detector are shown. The corresponding cumulative integrals of these pulses are also shown in Fig. 1. The baseline, calculated as an average of 600 samples preceding the leading edge of each pulse, was subtracted to avoid effects of voltage drift. The PMT and SiPM voltages were chosen to ensure that the pulses peaked between $20 \mathrm{mV}$ and $900 \mathrm{mV}$ in order to avoid voltage saturation of the digitizer. Pulses typically drop to $1 \%$ of their peak value after $100 \mathrm{~ns}$ for the PMT and $800 \mathrm{~ns}$ for the SiPM. The uncertainty introduced by digitization is below $1 \mathrm{mV}$ in both cases. A consistent pulse start time $t_{0}$ was defined in a way that minimized effects due to variations in pulse noise, baseline, amplitude and shape through the application to each pulse of a softwareimplemented digital constant fraction discriminator filter. ${ }^{12}$

For each recorded event, a parameter $Q_{L}$ was calculated by integrating the digitized pulses over a time interval $\left(t_{L}\right)$. The integration starts from a time $t_{0}$ that captures as much of the entire pulse as possible while still avoiding saturation of the available output transfer rate of the digitizer. Typical values for $t_{L}$ are $250 \mathrm{~ns}$ for the PMT and $1200 \mathrm{~ns}$ for the SiPM. The gamma rays from ${ }^{22} \mathrm{Na},{ }^{137} \mathrm{Cs},{ }^{207} \mathrm{Bi}$ and $\mathrm{AmBe}$ were used for scaling $Q_{L}$ to a light output parameter $L$ in units of $\mathrm{MeV}$ electron equivalent $\left(\mathrm{MeV}_{\mathrm{ee}}\right)$. The relationship between $Q_{L}$ and $L$ was found to be linear over the energy range of the calibration sources, and was assumed to be linear over the energy range of the measurements. Utilizing two SiPMs for the $6 \times 6 \times 50 \mathrm{~mm}^{3}$ scintillator led to significant improvements in energy resolution, pulse shape discrimination and linearity at higher $L$.

A second parameter $Q_{S}$ was calculated by integrating the pulse over a shorter time interval $\left(t_{S}\right)$, typically around $30 \mathrm{~ns}$ for the PMT and $60 \mathrm{~ns}$ for the SiPM. A pulse shape parameter $S$ was then defined as

$$
S=k \frac{Q_{S}}{Q_{L}}+C,
$$

which is the basis of a charge comparison method of PSD. ${ }^{12}$ Constants $k$ and $C$ were chosen in order to appropriately scale and offset $S$. Signals arising from gamma ray interactions with the detector have a reduced slow decay-component in comparison to 


\section{A. Buffler et al.}

those arising from neutron interactions. Therefore, a larger proportion of light output occurs within the shorter time interval in gamma ray events, leading to higher values of $S$ when compared to neutron events of a similar light output. Statistical fluctuations in pulse shape lead to Gaussian distributions of $S$ for gamma ray and neutron events with equal values of $Q_{L}$.
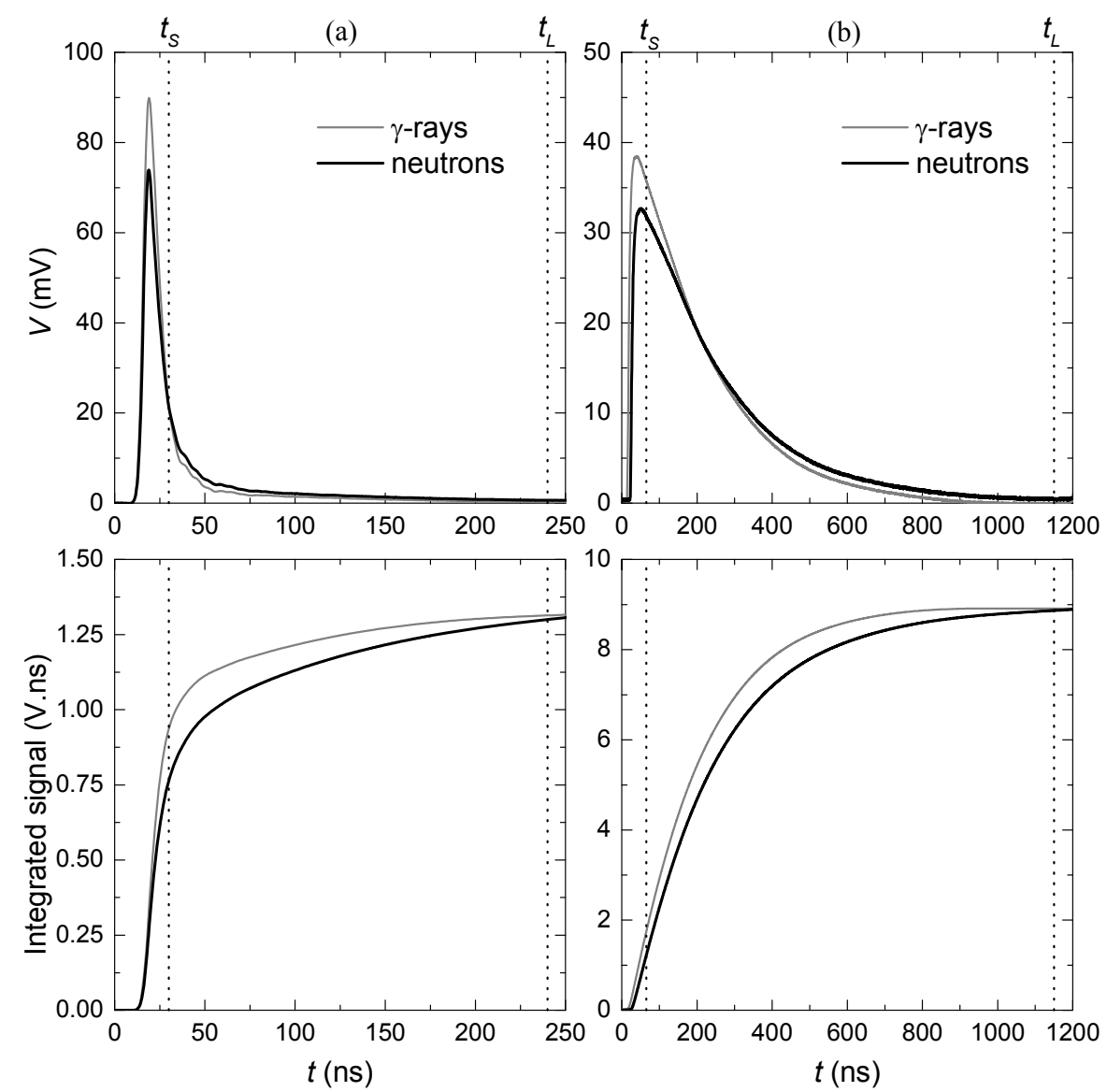

Fig. 1. Typical digitized pulses (above) and pulse integrals (below) for events arising from neutron and gamma ray interactions in the EJ299-33 scintillators of dimensions: (a) $50 \mathrm{~mm}$ diameter $\times 50 \mathrm{~mm}$ and (b) $6 \times 6 \times 50 \mathrm{~mm}^{3}$. Integration times $t_{S}$ and $t_{L}$ are indicated.

\section{Measurements with the AmBe Source}

Figure 2 shows distributions of events as a function of parameters $L$ and $S$ for two typical runs with the AmBe source. The loci associated with recoiling electrons (e) from Compton scattering events ("gamma rays") and protons (p) recoiling from n-p elastic scattering ("neutrons") are well separated over the full range of $L$. The gamma ray events result mainly from the detection of $4.43 \mathrm{MeV}$ gamma rays from the de-excitation of ${ }^{12} \mathrm{C}$ 
in the AmBe source. The "soft" slanted threshold at low $L$ is a result of the dependence of the trigger of the digitizer module on the signal height, rather than the magnitude of $Q_{L}$. This leads to a lower threshold for gamma ray events since these pulses have a shorter decay time. The dashed lines in Fig. 2 indicate the cut used to select events in the range $L=1.9$ to $2.1 \mathrm{MeV}_{\text {ee }}$, which are projected onto the $S$-axis in Figure 3(a). A figure of merit $(\mathrm{FoM})$ is a useful means to provide a quantitative measurement of the separation between the distributions of $S$ for two pulse classes. ${ }^{13}$ If these distributions are Gaussian in form, it is reasonable to define a FoM in terms of the mean $\mu$ and full width at half maximum (FWHM) of the two distributions in the form

$$
\mathrm{FoM}=\frac{\left|\mu_{e}-\mu_{p}\right|}{\mathrm{FWHM}_{e}+\mathrm{FWHM}_{p}} .
$$

The FoM values for the distributions shown in Fig. 2 are displayed in Fig. 3b. A short integration time $t_{S}$ was chosen in order optimize the FoM, with a typical value of $35 \mathrm{~ns}$ for the PMT-based detector and $75 \mathrm{~ns}$ for the SiPM-based detector. The FoM is considerably higher for the SiPM-based detector across the light output range with a FoM of 1.0 at $0.25 \mathrm{MeV}_{\text {ee }}$.

(a)

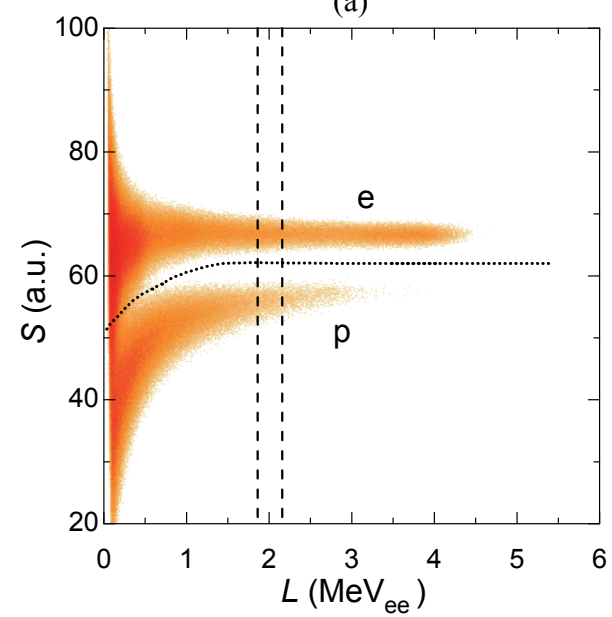

(b)

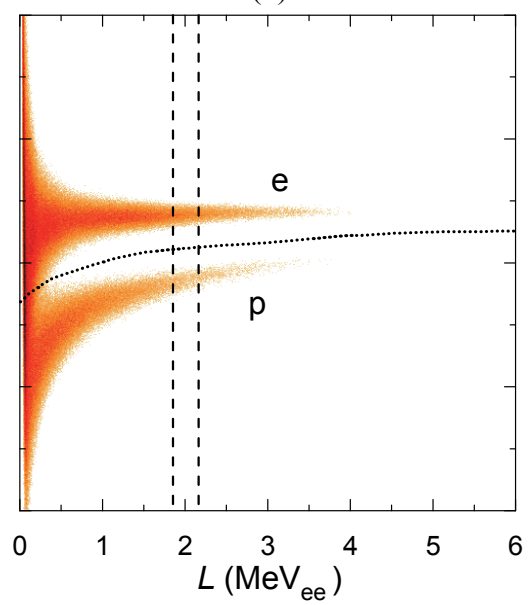

Fig. 2. Counts as a function of light output parameter $L$ and pulse shape parameter $S$ for events induced by neurons and gamma rays from an AmBe source in the EJ299-33 scintillators of dimension (a) $50 \mathrm{~mm}$ diameter $\times 50 \mathrm{~mm}$ and (b) $6 \times 6 \times 50 \mathrm{~mm}^{3}$. Loci associated with recoiling protons (p) and electrons (e) are identified. The dotted lines indicate the cuts used to separate neutron and gamma ray events and the dashed lines the cuts used to select events in the range $L=1.9-2.1 \mathrm{MeV}$. 
(a)

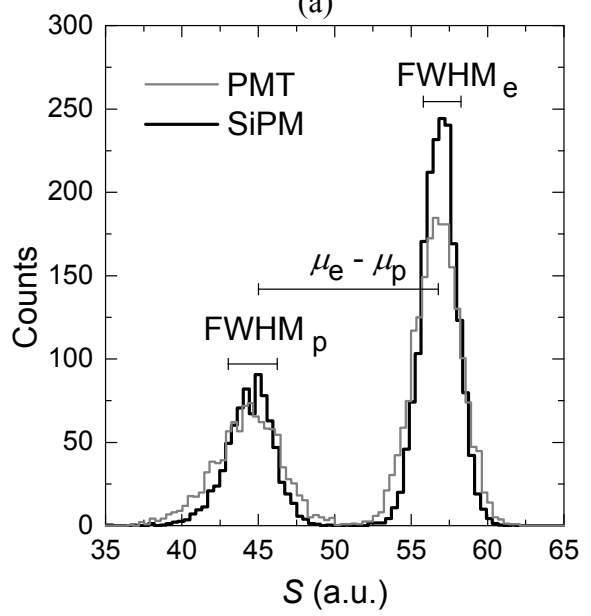

(b)

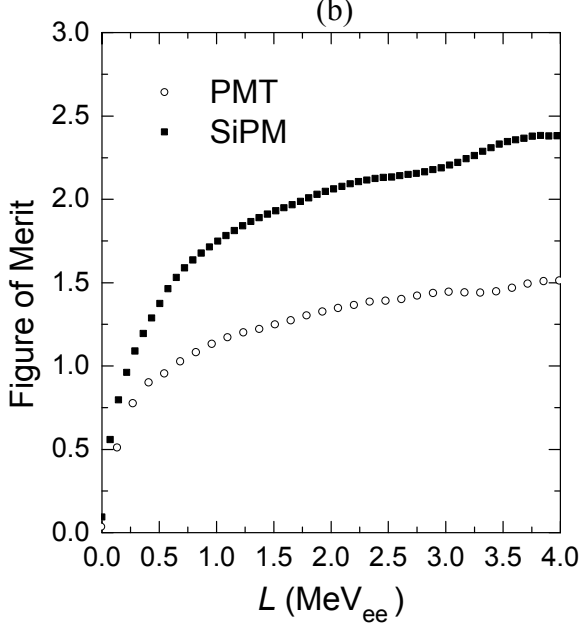

Fig. 3. (a) Counts versus shape parameter $S$ for events in the range of $L=1.9-2.1 \mathrm{MeV}_{\text {ee }}$ from a runs from runs with the Am-Be source, for the EJ299-33 scintillators of dimension $50 \mathrm{~mm}$ diameter $\times 50 \mathrm{~mm}(\mathrm{PMT})$ and $6 \times 6 \times 50 \mathrm{~mm}^{3}(\mathrm{SiPM})$. (b) Figure of merit versus $L$ for the same detectors.

A simulation of the $6 \times 6 \times 50 \mathrm{~mm}^{3}$ scintillator, together with light collection in the SiPMs, was constructed using the GEANT4 Monte-Carlo package. ${ }^{14}$ Light output relationships were adapted from Refs. 10 and 15, while energy resolution parameters were chosen to fit measured spectra. The simulation included a correction for the effect of saturation in the SiPMs, due to the finite number of pixels. Figure 4a shows a comparison between the measured and simulated spectra of a ${ }^{137} \mathrm{Cs}$ gamma ray calibration source. The discrepancy at lower pulse height is associated with gamma rays scattered off surrounding materials before entering the detector, an effect not fully incorporated in the simulation. A neutron response matrix was then created by simulating the response of the detector to monoenergetic neutrons up to $12 \mathrm{MeV}$, in steps of 0.2 $\mathrm{MeV}$. An illustrative sample of simulated line shapes is shown in Fig. 4b.

In order to evaluate the capability of the detector as a neutron spectrometer, spectrum unfolding was performed using the HEPRO package ${ }^{16,17}$ which has previously been used to successfully unfold light output spectra of EJ299-33 at higher energies. ${ }^{18}$ An AmBe neutron source was utilized, and the measured light output spectrum was unfolded using the simulated response matrix. The unfolded energy spectrum is compared with the reference spectrum ${ }^{19}$ in Fig. 5a. The unfolded spectrum matches the reference spectrum well with deviations below $3 \mathrm{MeV}$ most likely due to the interaction of the detector with neutrons scattered off surrounding material. Figure $5 \mathrm{~b}$ shows the measured and re-folded light output spectra. The two spectra are in excellent agreement across the relevant light output range, with a coefficient of determination of $R^{2}=0.998$. 
(a)

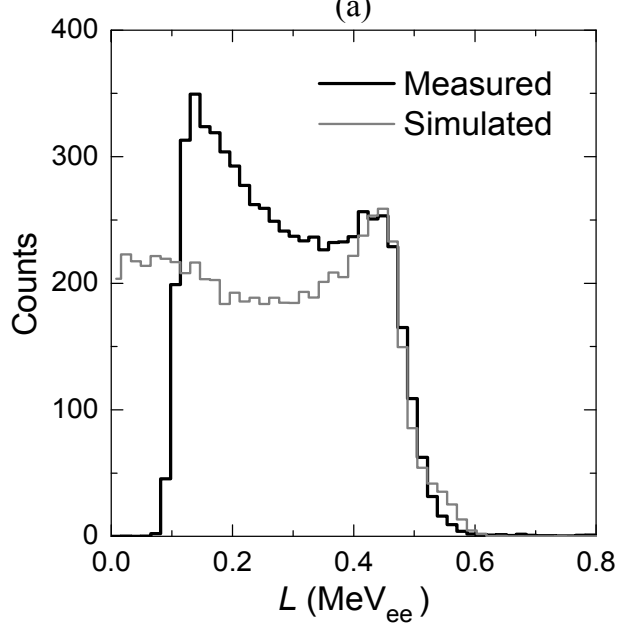

(b)

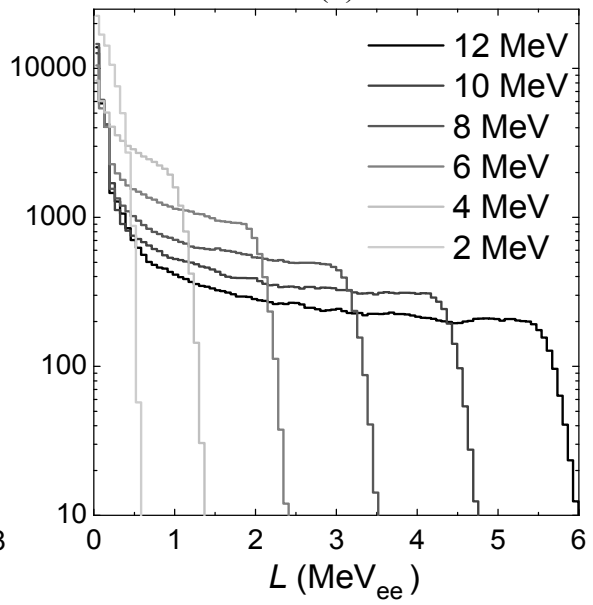

Fig. 4. (a) Line shape for the $6 \times 6 \times 50 \mathrm{~mm}^{3}$ scintillator (SiPM) measured for the $0.662 \mathrm{MeV}$ gamma ray from ${ }^{137} \mathrm{Cs}$ and simulated using GEANT4. ${ }^{14}$ (b) Neutron line shapes for the $6 \times 6 \times 50 \mathrm{~mm}^{3}$ scintillator (SiPM) simulated using GEANT4.
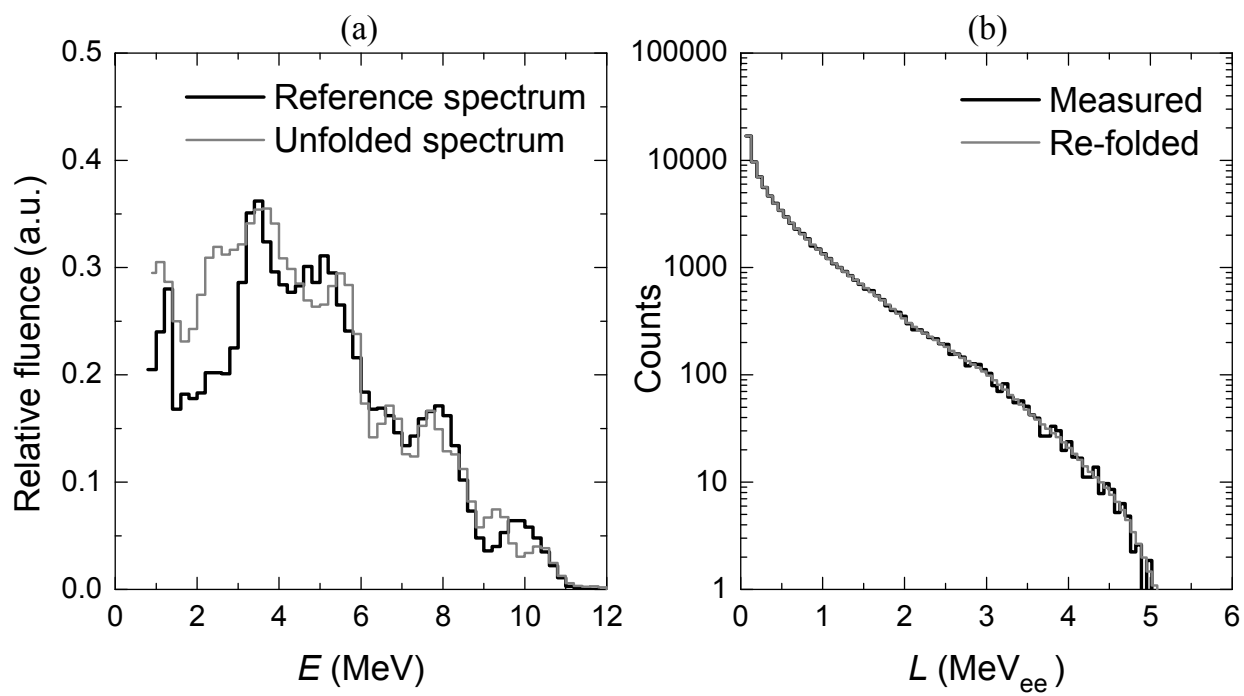

Fig. 5. (a) Unfolded neutron energy spectrum for neutrons from an AmBe source for the EJ299-33 detector of dimension $6 \times 6 \times 50 \mathrm{~mm}^{3}$ (SiPM) compared to the recommended spectrum ${ }^{19}$. (b) Re-folded light output spectrum, compared to the direct measurement.

\section{Measurements with a $140 \mathrm{MeV}$ Proton Beam}

The Separated-Sector Cyclotron of the iThemba LABS accelerates protons in the energy range from 20-200 MeV. A beam-pulse selector can suppress a chosen fraction of proton bunches to enlarge the time interval between pulses, allowing time of flight (ToF) 


\section{A. Buffler et al.}

measurements to be performed ${ }^{20}$ with less interference from earlier or later pulses. Typical currents for $100 \mathrm{MeV}$ are about $5 \mathrm{~mA}$ in unselected mode and $500 \mathrm{nA}$ at the repetition rate of $2.5 \mathrm{MHz}$. The ${ }^{7} \mathrm{Li}(\mathrm{p}, \mathrm{n}){ }^{7} \mathrm{Be}$ reaction was employed to produce quasimonoenergetic neutron beams because this reaction only induces the transition to the ground state and the first excited state of ${ }^{7} \mathrm{Be}$, with all higher levels being unstable. A quasi-monoenergetic neutron emission, which is strongly forward peaked, is obtained in this way. ${ }^{20}$ A $2.00 \mathrm{~m}$ thick steel collimator with openings at $0^{\circ}$ and $16^{\circ}$ shapes $8 \times 8 \mathrm{~cm}^{2}$ beams at $6.00 \mathrm{~m}$. The collimator is lined on the outside by a layer of borated wax and polyethylene. A cleaning magnet deflects proton beams into the well-shielded beam stop where a Faraday cup measures the beam current. In the present work, a proton energy of $140 \mathrm{MeV}$ was directed onto an $8 \mathrm{~mm}$ thick natural $\mathrm{Li}$ target, and the detector was mounted coaxially at $6.00 \mathrm{~m}$ from the target.

Figure 6a shows the distribution of events as a function of $Q_{L}$ and $T$ for measurements made using the $50 \mathrm{~mm}$ cylindrical EJ299-33 detector. The projection of the data onto the $T$-axis is shown in Fig. $6 \mathrm{~b}$. The sharp peak at $T=25 \mathrm{~ns}(\mathrm{FWHM}<1.3$ $\mathrm{ns}$ ) is associated with gamma rays produced in the Li target, while the uniform timeindependent distribution with $Q_{L}<12500$ is associated with background gamma rays in the experimental hall. The distribution at $T=50 \mathrm{~ns}$ is associated with direct reaction transitions mainly to the ground state of ${ }^{7} \mathrm{Be}$ and to the unresolved first excited state.

(a)

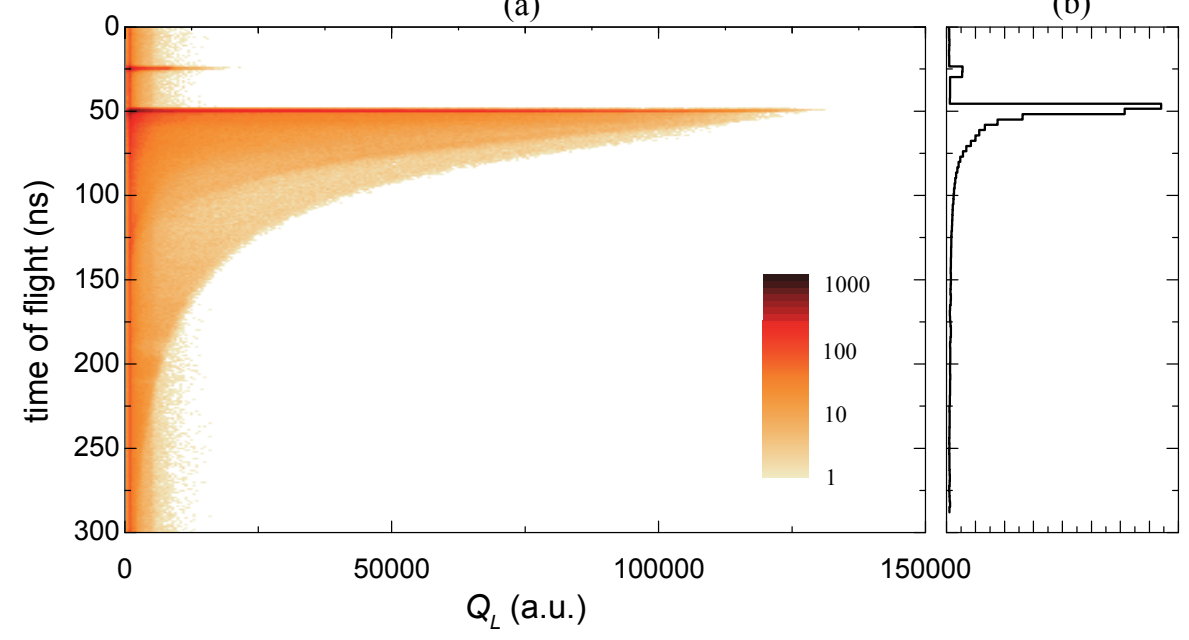

Fig. 6. (a) Counts as a function of $Q_{L}$ and time-of-flight parameter $T$ for the cylindrical EJ299-33 scintillator of dimension $50 \mathrm{~mm}$ diameter $\times 50 \mathrm{~mm}(\mathrm{PMT})$, from measurements made using a $140 \mathrm{MeV}$ proton beam irradiating an $8.0 \mathrm{~mm}$ Li target, at a distance of $6.00 \mathrm{~m}$ from the target. (b) Projection of the events onto the $T$-axis. 
(a)

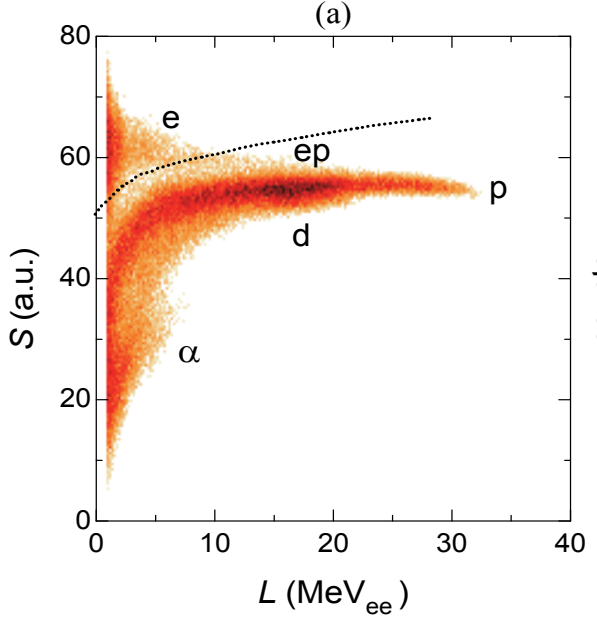

(b)

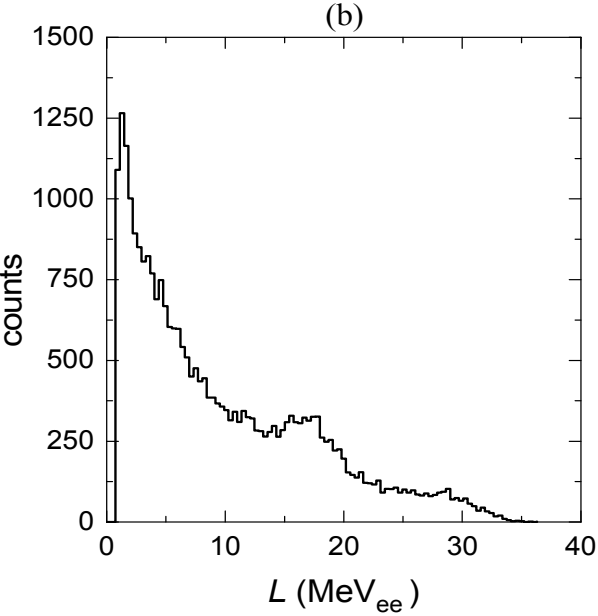

Fig. 7. (a) Counts as a function of light output parameter $L$ and pulse shape parameter $S$ for events in theEJ29933 scintillator of dimension $50 \mathrm{~mm}$ diameter $\times 50 \mathrm{~mm}$ (PMT), when exposed to neutrons and gamma rays produced by the irradiation of a Li target by a proton beam of energy $140 \mathrm{MeV}$, selecting events with $47.5<E$ $<52.5 \mathrm{MeV}$ by time-of-flight. Loci associated with recoiling electrons (e), protons (p), escaping protons (ep), deuterons (d) and alpha particles $(\alpha)$ are indicated. The dotted line indicates the cut used to separate gamma ray events from neutron events, which are projected into the $L$-axis in (b).

Figure 7a shows the distribution of events as a function of $L$ and $S$, selecting events across a $5 \mathrm{MeV}$ energy range via time-of-flight, centered at $50 \mathrm{MeV}$. Loci attributed to particles released by $n-p$ elastic scattering or by $n-C$ interactions in the scintillator are identified as: protons (p); deuterons (d); tritons (t) and alpha particles $(\alpha)$. Locus (e) is associated with recoiling electrons from gamma ray interactions, and locus (ep) with protons that escape from the detector before depositing their Bragg peak, reducing the proportion of $Q_{L}$ to $Q_{S}$. The loci overlap somewhat when compared to similar measurements with EJ3012 ${ }^{12}$ due to the poorer PSD capability of EJ299-33. The events associated with gamma rays from the target, however, can be efficiently excluded by the cut shown in Fig. 7a. The projection of the events onto the $L$-axis thus constitutes the response of the detector to $50 \mathrm{MeV}$ neutrons only and is shown in Fig. 7b. Line shapes measured in this way have been used to demonstrate the unfolding capability of EJ299-33 for neutron fields between 10 and $100 \mathrm{MeV} .{ }^{18}$

\section{Discussion and Conclusion}

Progress on the development of a new compact neutron spectrometer is reported. The device is based on EJ299-33 plastic scintillator, silicon photomultipliers, and digital pulse processing electronics, including the implementation of pulse shape discrimination to separate signals associated with neutrons and gamma rays. We have shown that a scintillator of dimension $6 \times 6 \times 50 \mathrm{~mm}^{3}$ (coupled to two SiPMs) can be employed to produce an excellent measurement of the energy spectrum of the neutrons produced by an 
AmBe source through the unfolding of a measured pulse height spectrum using a neutron response matrix for the detector calculated via GEANT4. Furthermore, the detector will be able to measure neutrons up to $100 \mathrm{MeV}$ by the extension of the response matrix through measurements using quasi-monoenergetic neutron beams, selected by time-offlight. At these higher energies, calculations of response functions are unreliable due to discrepancies in the cross sections for the many $n-C$ reaction channels that open up. We have shown that measured monoenergetic response functions can be produced in this way and that reliable unfolding is achievable using a larger scintillator and photomultiplier tube. ${ }^{18}$ The next phase of the work will focus on extending the compact spectrometer described here to $100 \mathrm{MeV}$ through further measurements at the neutron beam facility of iThemba LABS.

\section{Acknowledgments}

The authors would like to thank the National Research Foundation (South Africa) for financial support.

\section{References}

1. P. Goldhagen, J. M. Clem and J. W. Wilson, Radiat. Protect. Dosim. 110, 387 (2004).

2. G. T. Wright, Proc. Phys. Soc. Section B69 3, 358 (1956).

3. F. D. Brooks, Nucl. Instr. Meth. 162, 477 (1979).

4. N. Zaitsev et al., Nucl. Instr. Meth. A668, 88 (2012).

5. Eljen technology, EJ-299-33 plastic scintillator [Online]. Available: http://www.eljentechnology.com/index.php/products/plastic-scintillators/114-ej-299-33.

6. Y. Kaschuck and B. Esposito, Nucl. Instr. Meth. A551, 420 (2005).

7. K. A. A. Gamage, M. J. Joyce and N. P. Hawkes, Nucl. Instr. Meth. A642, 78 (2011).

8. C. C. Lawrence et al., Nucl. Instr. Meth. A759, 16 (2014).

9. S. Nyibule, E. Henry and W. U. Schröder et al., Nucl. Instr. Meth. A728, 36 (2013).

10. R. Preston, J. E. Eberhardt, R. Bencardino and J. Tickner, in Nuclear Science Symposium and Medical Imaging Conference (NSS/MIC) (IEEE, 2012), p. 462.

11. SensL, C-Series silicon photomultipliers [Online]. Available: http://www.sensl.com/downloads/ds/DS-MicroCseries.pdf

12. A. C. Comrie, A. Buffler, F. D. Smit and H. J. Wörtche, Nucl. Instr. Meth. A772, 43 (2015).

13. C.E. Brient, C. E. Nelson and R. L. Young. Nucl. Instr. Meth. 98, 329 (1972).

14. S. Agostinelli, et al., Nucl. Instr. Meth. A506, 250 (2003).

15. C. C. Lawrence et al., Nucl. Instr. Meth. A759, 16 (2014).

16. M. Matzke, Unfolding of Pulse Height Spectra: The HEPRO Program System, PhysikalischTechnische Bundesanstalt Report PTB-N-19 (1994).

17. H. Klein and S. Neumann, Nucl. Instr. Meth. A476, 132 (2002).

18. A. Buffler, A. C. Comrie, F. D. Smit, and H. J. Wörtche, Neutron Spectrometry with EJ299-33 Plastic Scintillator for $E_{n}=10-100 \mathrm{MeV}$, to appear in IEEE Trans. Nucl. Sci.

19. I. Kawrakow and D. W. O. Rogers, The EGSnrc Code System: Monte Carlo Simulation of Electron and Photon Transport, NRCC Report PIRS-701 (2000).

20. M. Mosconi et al., Rad. Meas. 45, 1342 (2010). 